\title{
Chaos and Poststructuralism
}

\begin{abstract}
A $T$ the same time that new concepts of chaos and randomness are changing the way scientists think about informational systems, they are also affecting the way literary critics write about texts. The major impetus for this revision has come from poststructuralism, especially deconstruction. Paul de Man, for example, sees deconstruction as warning us that "nothing, whether deed, word, thought or text, ever happens in relation, positive or negative, to anything that precedes, follows or exists elsewhere, but only as a random event whose power . . . is due to the randomness of its occurrence" ( r979a: 69). As the text is opened to an infinitude of readings and as meaning becomes indeterminate or disappears altogether, chaos apparently reigns supreme. In this extreme form, deconstruction seems to have gone beyond the premises that make science possible.

Yet Geoffrey Hartman (r976), confronted with the "tangled, contaminated, displaced, deceptive" text of Derrida's Glas, speculates that deconstruction is opposed to more traditional, humanistic readings because it is more scientific. "The result for our time [of deconstruction in general and Glas in particular] may be a factional split between simplifying types of reading that call themselves humanistic and indefinitizing kinds that call themselves scientific," Hartman writes (p. I 83). He is correct, perhaps in a sense he did not intend, in linking deconstruction's “indefinitizing” strategies with science.


Deconstruction shares with chaos theory the desire to breach the boundaries of classical systems by opening them to a new kind of analysis in which information is created rather than conserved. Delighting in the increased complexity that results from this "scientific" process, both discourses invert traditional priorities: chaos is deemed to be more fecund than order, uncertainty is privileged above predictability, and fragmentation is seen as the reality that arbitrary definitions of closure would deny.

In this chapter I draw parallels between poststructuralist philosophy and scientific attitudes toward chaos, then discuss particular interpretive strategies employed by Jacques Derrida, Roland Barthes, and Michel Serres. In these analyses poststructuralism is my subject, not my method. Whereas poststructuralist analyses commonly operate upon texts to open within them fissures, discrepancies, and inversions, my analyses operate upon poststructuralist texts to show that they share with modern science assumptions and methodologies that can hardly be explained without the assumption that both are part of a common episteme. My approach is constructive rather than deconstructive. It seeks to delineate an ecology of ideas, to see similarities between scientific and literary theories as interrelated propositions that appear in separate discourses concurrently because they are responses compatible with the cultural environment. ${ }^{.}$This approach prevails especially in the section that takes Derrida and nonlinear dynamics as its focus.

An ecological approach is not, however, able to explain the very real differences between scientific models and critical theory. To elucidate how different evaluations can emerge from similar premises, I turn to an economic model. Information theory and poststructuralism concur in assigning a positive value to chaos. But where scientists see chaos as the source of order, poststructuralists appropriate it to subvert order. Thus the scientific response appears fundamentally conservative, whereas the poststructuralist stance styles itself radical. When institutional practices within the two disciplines are taken into account, however, both responses appear equally conservative, serving to perpetuate rather than challenge the economic in-

'The term "ecology of ideas" alludes to Gregory Bateson's Steps to an Ecology of Mind (San Francisco: Chandler, 1972). I am indebted to Bateson's illuminating analyses of communication dialectics throughout this chapter. 
frastructures of their disciplines. Representative texts discussed in this section are Barthes's $S / Z$ and Shannon and Weaver's Mathematical Theory of Communication.

The final section explores the synthesis of science and literature in the work of Michel Serres. A key concept for him is equivocation, the term that Shannon used to describe what is added to or subtracted from information as it passes through a noisy channel. Equivocation's inherent ambiguity-does noise add to information or subtract from it? - is present on a deep level in Serres's project. Demonstrating a strong proclivity toward order at the same time that it celebrates disorder, his work simultaneously tries to liberate noise at specific sites and to suppress it in global theories. The multiple levels at which these equivocations work within his discourse suggest that a theorist who locates himself at the crossroads of disciplines is able to maintain this position because he employs linguistic and conceptual structures capable of mediating between different disciplinary economies.

Throughout the three sections, I am concerned with rifts as well as convergences, differences, and similarities. Yet the final impulse is to show that both scientific and literary discourses are being distinctively shaped by a reevaluation of chaos. It is this vision that defines the contemporary episteme and differentiates it from the modernist era.

\section{Ecology}

As is well known, deconstruction grew out of developments in linguistics, particularly the Saussurean distinction between the signifier and the signified..$^{2}$ As long as this distinction is accepted as valid, we remain in the realm of semiotics rather than deconstruction. As Saussure recognized, however, the signified is a concept rather than an object. It is a small step to consider the signified as another signifier in turn; this step carries us out of semiotics into deconstruction. For if the signified is another signifier, then its signified is also a

'For an overview see Jonathan Culler, Ferdinand de Saussure (New York: Penguin, 1977) and The Pursuit of Signs: Semiotics, Literature, Deconstruction (Ithaca: Cornell University Press, 1981 ). 
signifier, and we are caught in an endless chain of signifiers whose meanings, if they can be said to exist, are indeterminate.

Saussure initiated semiotics by proposing that the proper study for linguistics was la langue rather than la parole, the system of language rather than words, and by showing that relations within la langue could be specified only as a series of differences. The analogous movement within science came with Shannon's theory of information. Saussure's theory separated sign from referent; Shannon's theory separated information from meaning. In Shannon's equations, the informational probability of an element can be calculated only with reference to the ensemble from which it is drawn, that is, not absolutely but through a series of differences. ${ }^{3}$ This move allows the information content of a message to be quantified regardless of its context or meaning. The inward-turning structures of Saussurean linguistics and information theory are not arbitrary theoretical choices. In the absence of external reference, these theories could be defined only internally. (I explore the cultural implications of these isomorphic strategies in chapter ro, arguing that they both reflect and reinscribe the postmodern condition.)

With deconstruction, the deeper implications of defining language and texts through their internal relations became explicit. Derrida's Of Grammatology (1967; English translation 1976) announced the birth of this deconstructive "monstrosity" (1976: 5). Until now, Derrida says, Western thought has persisted in believing in a Logos capable of revealing immediate truth. Speech is privileged because it is the embodiment of Logos, the word that is also presence. Writing, by contrast, is belated, a fall from presence into absence, the signifier of a signifier, inferior because twice mediated. These assumptions are inverted in Of Grammatology, which proclaims the beginning of a "grammatological" epoch in which writing is privileged over speech.

At the center of grammatology is Derrida's redefinition of writing. In the grammatological view, writing ("écriture") is not merely written marks but any signifying practice that endures through time and functions to divide the world into self and other. This view of writ-

${ }^{3}$ For a clear explanation of how difference enters into Shannon's theory, see Norman Abramson, Information Theory and Coding (New York: McGraw-Hill, 1963), pp. $\mathrm{I}-44$. 
ing was apparently inspired by Freud's short essay "The Mystic Writing Pad," in which he likened the psyche to the child's toy that in this country is known as a magic slate. ${ }^{4}$ The conscious mind, Freud suggested, is like the transculent sheet on which the child marks with a pointed stick. The marks are visible because the sheet is momentarily pressed into a wax base underneath. When the sheet is raised, the marks disappear. But they have not entirely vanished; their impressions linger in the wax base. The earliest experiences of childhood, Freud argued, are like the marks on the sheet, disappearing from the conscious mind as the child matures. But they leave traces in the deeper strata of the psyche, just as the marks leave traces in the wax. For Derrida, writing includes not only marks on the page but these deeper traces within the psyche. Residing at a deeper level than words can reach, the Derridean trace remains inaccessible to direct verbalization. It is "always already" present, the elusive and ineffable difference from which all subsequent inscription derives. In this sense writing, as the mark of difference, not only precedes speech but actually brings it into being.

By making the trace inaccessible and indeed unknowable, Derrida opens writing to radical indeterminacy. In contrast to speech, writing operates according not just to Saussurean difference but to Derridean "différance," a neologism that in French combines "to differ" with "to defer" (Derrida, I 968). The "always already" formula implies that there is no origin, that the very idea of origin is an illusion. Différance acknowledges a before and after - that is, a constituting difference-but defers indefinitely the moment when this split occurred. No matter how far back we go into signification, we never come upon the originary difference that could act as ground for the, chain of signifiers.

After writing Of Grammatology, Derrida moved on to other metaphors to uncover fissures within Western metaphysics-the pharmakon, the postcard, the hymen-presumably to avoid having his project reify into a methodology that could become a metaphysics in turn. Although there are suggestive parallels with the new paradigms

${ }^{4} \mathrm{My}$ discussion of the trace is conjectural, as it is important to Derrida not to define it. Derrida discusses Freud's essay and its implications for deconstruction in "Freud and the Scene of Writing," in Writing and Difference, trans. Alan Bass, pp. 196-23 I (Chicago: University of Chicago Press, 1978). 
in much of this later work as well, the resonances are perhaps strongest in the early work in Of Grammatology, which also corresponds most closely in time to the first wave of important discoveries in chaos theory. I therefore will concentrate on this work, recognizing that it is only one phase of Derrida's continuing development of deconstructive methodologies.

That there is a methodology at work is a disputed claim, for positing a method is a first step toward establishing an orthodoxy that could quickly defeat the intent to unravel and undermine. Derrida himself is extremely canny about recognizing and avoiding this danger; even as early as Of Grammatology, he warned that his readings were too "exorbitant" and "excessive" to fit into any standard methodology (I 976: I 57-164). Nevertheless, one can scarcely fail to notice that deconstructive analyses tend to follow a predictable pattern, especially in the hands of disciples less adroit than Derrida in avoiding the dangers of reification. The method appears in paradigmatic form in Of Grammatology. First a received duality (speech/ writing) is destabilized by inversion; then the existence of a third term (the trace) is revealed whose nature is undecidable because by definition it falls outside the realm of discourse. The validity of the speech/writing duality is thereby drawn into question, but no new dialectic emerges to take its place because the emergence of a dialectic would depend upon being able to decide what the trace is. In the grammatological approach, the original concepts do not entirely disappear but are put under erasure ("sous rature"). Seen and not seen, absent and yet present, they function as reminders that the old meanings are gone and as remainders that keep new ones from forming. Thus Derrida does not intend simply to replace one set of priorities with another. Rather he attempts to undermine the very process by which meaning is constituted.

The vertigo characteristic of deconstruction appears when we realize that texts are always already open to infinite dissemination. Far from being ordered sets of words bounded by book covers, they are reservoirs of chaos. Derrida initiates us into this moment in Of Grammatology through his concept of iteration. Any word, he argues, acquires a slightly different meaning each time it appears in a new context. Moreover, the boundary between text and context is not fixed. Infinite contexts invade and permeate the text, regardless 
of chronology or authorial intention. Hamlet, for example, influences our reading of Rosencrantz and Guildenstern Are Dead; but Rosencrantz and Guildenstern Are Dead also influences our reading of Hamlet. The permeation of any text by an indefinite and potentially infinite number of other texts implies that meaning is always already indeterminate. Because all texts are necessarily constructed through iteration (that is, through the incremental repetition of words in slightly displaced contexts), indeterminacy inheres in writing's very essence.

We can see iteration at work in the dense, highly repetitive analysis of Rousseau that occupies the last half of the Grammatology. Rousseau is well suited to Derrida's deconstructive project because his thought is expressed through a series of hierarchical dualities: nature/culture, animal/human, speech/writing. For Rousseau, the first term of each of these dualities is privileged. The second term is belated, contaminated, a fall from the "pure" first term. His announced aim is to correct modern decadence by returning to the originary first term, rejecting culture for nature, writing for speech, and so forth.

Through a rigorous reading of Rousseau's texts, Derrida shows that this attempt at purification is fundamentally misguided because the idea of origin is an illusion. The demonstration concentrates on the "supplément," a word that Rousseau uses in the Confessions as a euphemism for masturbation. Sex is natural, good, healthy; but tormented by fear of women and venereal disease, Rousseau continually finds it necessary to resort to the supplement. Derrida shows that a similar dialectic emerges with each set of Rousseau's dualities (1976: 14r-164). Rousseau denounces writing but does so by writing texts; he embraces nature but finds that its deficiencies require the education he advocates in Emile, and so forth. To supplement something implies that the original is already full and self-sufficient,

5For a discussion of iteration, see Derrida, Of Grammatology (1976), pp. 157I62. The concept of iterability also plays a prominent role in "Signature Event Context" (1977b) and in Derrida's subsequent deconstruction of John Searle's "Reply" to "Signature Event Context" in "Limited Inc abc ..." (I 977a). A fuller analysis of how iteration enters into the Derrida-Searle exchange is given in N. Katherine Hayles, "Disciplinarity and the University: What It Keeps Us from Seeing," in The Nature of the University, ed. Peter Shane (Iowa City: University of Iowa Press, forthcoming). 
in contrast to the supplementary material, which comes after and is superfluous. Yet in each case the first term- "nature," for example-is "naturally" deficient, so that the supplement is indispensible. In what sense then is the supplement more "unnatural" than nature? Through this implicit contradiction, Derrida shows that the supplement is in fact what allows the privileged term to be constitu$t e d$. The originary precedence of the privileged term is revealed as an illusion, a myth or longing for origin rather than an origin as such.

According to Derrida, every text will have a concept that functions as the supplement does in Rousseau. The supplement (or its analogue) is, Derrida argues, a kind of fold in the text whose indeterminacy is revealed through repetition. ${ }^{6}$ In his view such a fold is necessarily present, because there must always be some means by which the text can constitute the differences that enable it to postulate meaning. The fold can be thought of as a way to create the illusion of origin. Once it is in place, all subsequent differences are declared to derive from the originary difference marked by the fold. When the text is "unfolded," this stratagem is revealed and the regulated exchanges between the alleged origin and subsequent differences that enable the text to operate will appear.

It is precisely this "unfolding" that iteration accomplishes. In Derrida's hands, repeating Rousseau's language with incremental differences becomes a way to unfold and make visible the inherent contradictions upon which the text's dialectic is based. This iterative procedure produces the undecidables that radically destabilize meaning. "It [is] certainly a production," Derrida writes, "because I do not simply repeat what Rousseau thought of [the supplement]. The concept of the supplement is a sort of blind spot in Rousseau's text. ... [The production of undecidables] is contained in the transformation of the language [that the text] designates, in the regulated exchanges between Rousseau and history. We know that these exchanges only take place by way of the language and the text" (1976: I63-I64). The goal of iteration is thus to make visible the lack of ground for the alleged originary difference, thus rendering all subsequent distinctions indeterminate.

"Derrida speaks of a "certain deconstruction which is also a traced path" (1976:162). 
Derrida's deconstructive methodology is strikingly similar to the mathematical techniques of chaos theory. Recall that Feigenbaum attributed the universal element in chaotic systems to the fact that they were generated from iterative functions (1980: 4-27). He showed that for certain functions, individual differences in the equations are overwhelmed as iteration proceeds, so that even though the systems become chaotic, they do so in predictable or regulated ways. Derrida claims that his iterative methodology is similarly regulated, in the sense that its production of undecidables is not a capricious exercise but a rigorous exposition of the text's inherent indeterminacies.

For both Derrida and Feigenbaum, iterative methodology is closely tied in with the concept of the fold. Feigenbaum showed that systems that make orderly transitions to chaos always have folds in their iterative paths (p. 9). Within the complex regions created by these folds, orbits wander in unpredictable ways. Where does this unpredictability come from? Since the iterative formulae and computer algorithms are perfectly deterministic, it could come only from the initial conditions. Iteration produces chaos because it magnifies and brings into view these initial uncertainties. Similarly, Derrida attributes textual indeterminacy to the inherent inability of linguistic systems to create an origin. In Derrida, "always already" marks the absence of an origin, just as inability to specify initial conditions with infinite accuracy does for Feigenbaum. Thus nonlinear dynamics and deconstruction share not just a general attitude toward chaos, but specific methodologies and assumptions.

There are, of course, also significant differences between them. Feigenbaum works with mathematical formulae that are capable of exact definition; Derrida is concerned with language, which is notoriously resistant to formalization. One measure of these differences is disagreement among deconstructionists and scientists on how extensive chaos is. For Derrida, textual chaos is always already in Rousseau and in every other text. Scientists, by contrast, acknowledge that ordered, predictable systems do exist, although they are not nearly so widespread as classical science had supposed. Feigenbaum, for example, takes for granted that only certain classes of iterative functions become chaotic. Moreover, he acknowledges that until very recently, virtually all scientific knowledge derived from the 
study of ordered systems (pp. 14-15). Whereas Of Grammatology forecasts an apocalyptic break with logocentrism (a position that Derrida was to modify and complicate in later work), scientists are likely to think of their work as a continuation of what has gone before. To a deconstructionist, a "recuperator" is beyond salvation; for most scientists recuperation is not even an issue, because they see their work as enhancing rather than discrediting traditional scientific paradigms. Gregory Chaitin is typical in his view that algorithmic complexity theory merely supplements classical probability theory rather than supplants it $(1975: 48)$. To the Derrida writing in Of Grammatology such a statement would itself invite deconstruction, for if the theory is supplemental it cannot be necessary, but since it is necessary, it cannot be supplemental. . . These differences are symptomatic of the different values the two camps place on chaos. For deconstructionists, chaos repudiates order; for scientists, chaos makes order possible.

These differences notwithstanding, Derridean deconstruction and nonlinear dynamics are strikingly parallel in a number of ways. They agree that bounded, deterministic systems can nevertheless be chaotic; they both employ iteration and emphasize folds; and they concur that originary or initial conditions cannot be specified exactly. Given Derrida's antipathy toward science (for him "objective" is a pejorative term), it is unlikely that his ideas are substantially indebted to scientific sources. It is equally difficult to believe that Feigenbaum, Ford, or Shaw has been influenced by Derrida. How to account for the similarities?

The two theories appear isomorphic not because they are derived from a common source or because they influenced each other, but because their central ideas form an interconnected network, each part of which leads to every other part. Feigenbaum worked on iterative functions in part because advances in computer technology made it easy to see how they performed over time. He was led to the idea of an unreliable origin not because he was interested in the questions of origin as such, but because it was the only place chaos could have entered the system. For Derrida, working in a field dominated by Hegel, Nietzsche, and Heidegger, the question of origin was highly charged. Once he postulated a lack of originary ground, iteration was an appropriate methodology because it has the effect 
of magnifying latent uncertainties. Thus Derrida and Feigenbaum entered the network at different points and for different reasons. But because chaos, iteration, and an unreliable origin form an interconnected system of ideas, the correlative concepts were brought into play once the implications of the original premise were explored. Deconstruction and nonlinear dynamics appear isomorphic, then, because the concepts with which they are concerned form an ecology of ideas.

A theoretical model for conceptual ecologies has been proposed by Stanislaw Lem (I98I), who suggests that they can be modeled as closed topological spaces. Within a given topology, only certain forms are possible. Others are prohibited by the overall spatial conformation. ${ }^{7}$ Not every possible form will be realized; particularities of history and personality determine which actually appear and which are repressed. All forms that are realized, however, are linked to each other through the common attributes that define the space.

Lem concedes that so many variables are at work within contemporary culture that it can never be rigorously modeled as a topological space. Topological models have, however, been successfully used in biology to predict (or, more accurately, to retrodict) what appendages will appear for a given environment and set of genomes. These programs are limited to retrodiction rather than prediction, for in a highly complex system it is not possible to predict exactly how the system will evolve because there are too many cusps where minute fluctuations cause wide variations in future behavior. ${ }^{8}$ Once one knows what has happened, however, it is possible to show that the realized forms are consistent with the system's topology. I conjecture that this is the case for nonlinear dynamics and deconstruction. They were not inevitable developments, but they were among the possible shapes that could evolve within postmodern culture. They are similar because they share in the constraints that define the overall topology.

${ }^{7}$ For an explanation of topology, see Bert Mendelson, Introduction to Topology (Boston: Allyn \& Bacon, r962).

${ }^{8}$ Catastrophe theory comes to very similar conclusions. See René Thom, Structural Stability and Morphogenesis: An Outline of a General Theory of Models, trans. D. H. Fowler (Reading: W. A. Benjamin, I 975). For discussions of catastrophe theory in relation to biology and linguistics, see René Thom, Modèles mathématiques de la morphogenèse (Paris: Union Général d'Editions, 1975). 
An advantage of the topological model is that it leaves room for other kinds of explanation. It designates which shapes are possible, but it does not say which will actually appear, or how their actualizations are affected by such factors as disciplinary traditions and individual desires. To understand why deconstruction and the science of chaos value disorder differently even though they model it in conceptually similar ways, we will need to consider what happens when ideas are expressed within the boundaries of specific disciplines and articulated through specific voices. Why, for example, does deconstruction want to break with the past, whereas nonlinear dynamics wants to emphasize historical continuity? How do isomorphic concepts change when they are expressed in the tropes and linguistic registers peculiar to an individual theorist? To explore these and other questions, we turn from ecology to economy and equivocation.

\section{Economy}

Warren Weaver remarks that Shannon's theory of information is powerful because of its economy of explanation (Shannon and Weaver, I 949: I I4-I I 5). Implicit in the comment is the assumption that the best theory is that which can explain the most diverse phenomena with the fewest principles. The tendency in science is to simplify, to reduce the many to the few-millions of chemicals to some hundred elements, for example, then a hundred elements to three atomic particles. When the atomic triad proliferated into hundreds of subatomic particles with the advent of high-energy physics, the scientific community was disturbed; economy had been violated. An intensive search was undertaken for a grand unified field theory. ${ }^{9}$ When its outlines began to emerge, its power was understood to reside in its ability to reduce an unruly mob to an elegantly simple number again, the four forces that govern the organization of matter. Shannon's theory of information falls squarely within this scientific tradition. His theorems are powerful in the sense that they reduce many different kinds of message signals to a few limit cases.

The passion for economical expression seems to have been a per-

${ }^{9}$ An overview of grand unified field theory can be found in Davies, I984. 
sonal as well as a professional aesthetic for Shannon. Colleagues recall him as a brilliant theorist who could not bear to relinquish his ideas for publication until they were expressed in the most economical form imaginable. In an anecdote recounted in Grammatical Man (Campbell, I982), Edward Moore recalls that Shannon "would let a piece of work sit for five years, thinking it needed to be improved, wondering if he had made the right choice of variable in this or that equation. Then, while he was still contemplating improvements, someone else would come out with a similar result that was correct, but so lacking in formal elegance that Shannon would have been ashamed to have done such a shoddy job."

In contrast to Shannon's dedication to economy is Roland Barthes's exuberant expansiveness in $S / Z$. $S / Z$ has a certain structuralist orientation in its impluse to classify and categorize Balzac's story Sarrasine in accordance with five discrete linguistic codes. Significantly, however, it uses these codes not to reduce but to expand Balzac's text. Thus it is even more poststructuralist, sharing with deconstruction the desire to increase message ambiguity as much as possible - for example, through split writing, dense syntax, elusive wordplay, and elliptical style. Barthes's project is ideological as well as aesthetic, for like Derrida he regards "correctness" as an illusion perpetrated by centrist philosophy to control texts, language, and power structures within society. In this respect information theory and $S / Z$ could scarcely be more different.

At first glance, it appears that the political stances implied by these different aesthetics are easily defined. Shannon's theory is conservative because it seeks to wrest order out of chaos, preserve message from noise, and guard correctness from contamination by error. $S / Z$ is radical because it seeks to liberate noise from message, release chaos from order, and overturn the hegemony of received interpretations. But because these theories do not act in isolation-because they are embedded within the economic infrastructures of their disciplines-their actual effects are more difficult to assess. I shall argue that when disciplinary contexts are taken into account, both theories are conservative, serving to perpetuate rather than challenge the traditions from which they come. First, however, it is necessary to look more closely at the transformations Barthes effects with Balzac's short story Sarrasine as he re-presents it in $S / Z$.

The radical stance that differentiates $S / Z$ from information theory 
is apparent throughout Barthes's text. Declaring that he is not interested in what Sarrasine means, Barthes distinguishes five codes at work within his "tutor text" and identifies them with so many "voices" speaking Sarrasine. He refuses to arrange them hierarchically in search of a total meaning. Moreover, he points out that even within one code disparate connotations are often at work, as if two voices were speaking at once over the same channel. These "equivocations," as Barthes calls them, are to be encouraged:

In relation to an ideally pure message (as in mathematics), the division of reception constitutes a "noise," it makes communication obscure, fallacious, hazardous: uncertain. Yet this noise, this uncertainty are emitted by the discourse with a view toward communication: they are given to the reader so that he may feed on them: what the reader reads is a countercommunication. [Barthes, I 974: I45]

Barthes concludes that "literatures are in fact arts of "noise" "and declares that this "defect in communication" is "what the reader consumes."

The equivocation in Barthes's own text comes into focus with the word "consumes." With his assertion that literature is noise, he situates his project in opposition to the information theory from which he takes his terms. "Consumes" is a pivotal word because it recalls the capitalistic context of Shannon's career. Working at Bell Laboratories, Shannon was necessarily concerned with commercial applications of his work. He devoted considerable attention to how to get a message through correctly (Shannon and Weaver, 1949: 3-93). As we saw in chapter 2, Shannon defined any deviation from the intended message as the equivocation. One of his most important theorems demonstrates that it is possible to reduce the equivocation to zero if the code is chosen correctly.

The equivocation that Shannon wants to eliminate, Barthes offers up for consumption. Moreover, he claims that the reader will find this extra information more delectable than the original message. The persistent use of oral imagery in Barthes's text creates a new context for consumption, associating it with gourmandizing rather than capitalism. In effect, Barthes replaces the commercial orientation that sees correctness as an important value with a sensuality that finds its most exquisite satisfaction in deviant orality. 
Within his discipline, however, Barthes's attitudes are not deviant; they merely express mainstream beliefs in a risqué fashion. For the economy of explanation that scientists regard with respect has long been viewed with suspicion in literary circles. Some critical methodologies have attempted a scientific economy of explanation-archetypal criticism and structuralism, for example-but they are the exceptions rather than the rule. In general the literary community favors convoluted explanations that expand the few to the many rather than economical explanations that reduce the many to the few. The phenomenon can, I believe, be understood in terms of the economic infrastructure of the discipline.

Before poststructuralism, literary criticism was confined to an accepted corpus of literary texts for its subject material. This body of texts remained essentially constant for decades, except for the influx provided by living writers; meanwhile, the academic literary establishment increased enormously. ${ }^{10}$ Even when the influx of new writers is taken into account, the domain of critical theory has been extremely restricted in comparison with the scientific domain, where advances in technology and instrumentation continually open new areas for research. Who among us has not known the fear of arriving too late, when the texts have all been used up? Perhaps the Bloomian concept of belatedness is not so much analysis as projection, for even more than literature, literary criticism has operated according to an economy of scarcity. Too many critics, too few texts.

Poststructuralism, especially deconstruction, overcomes this scarcity by showing how each text can be made into an infinite number of texts. Moreover, it actually converts scarcity to excess by proclaiming that theory's proper subject is not only literature but theory itself. ${ }^{11}$ In this sense, $S / Z$ represents less the cusp between structuralism and poststructuralism than a harbinger and consort of decon-

\footnotetext{
"This argument neglects, for simplicity, the movement to open literary study to noncanonical works. As this trend can also be understood as a response to disciplinary economy, it would not change the essence of the argument, although it would render it more complex.

"For an appraisal and reluctant acquiescence in this claim, see Geoffrey H. Hartman, "Crossing Over: Literary Commentary as Literature," Comparative Literature 28 (Summer 1976 ): $257^{-276}$.
} 
struction. By opening the text to dissemination and by blurring the distinction between "primary" and "secondary" works, deconstruction converts a closed system operating according to a scarcity economy into an open system based on autocatalysis. The more theory that is written, the more texts there are for theory to write about, because theory itself produces the texts that the next generation of theory will consume. As we saw in chapter 4 , physical systems that are autocatalyzing can spontaneously reorganize themselves at a higher level of complexity (Nicolis and Prigogine, 1977). Theorists, unlike molecules, are conscious of the systemic organization they help to build. The analogy is useful, however, because it suggests that increased complexity arises not merely because of a decision by an individual theorist but because the systemic economy demands it. Thus the increasing number of theoretical texts in literary criticism, as well as their tendency to organize themselves in increasingly complex ways, can be understood as responses to the discipline's systemic economy.

At this point the reader may object that my explanation is "scientific," in the pejorative sense of reducing complex phenomena to simplistic generalizations, when it relates deconstruction's popularity to its economic function within the discipline rather than to its intellectual and conceptual power. I do not dispute that deconstruction is powerful. I would point out, however, that what it means for a theory to be powerful varies from discipline to discipline. We cannot properly evaluate the claim for deconstruction's power without first inquiring into the disciplinary economy that gives meaning to this term. Such an inquiry demonstrates that the aesthetics of deconstruction and information theory are consistent with their respective disciplinary economies. Moreover, their contrasting views of what counts as message and as noise are also consistent with these economies.

It is no accident, then, that conceptually isomorphic theories take on different values when they are embedded in disciplinary matrices. Meaning is always dependent on context, and different disciplinary economies create significantly different contexts. These contexts are reinscribed in Shannon's and Barthes's diverging views of equivocation. They agree that all messages are mediated. However, Shannon sees this as a regrettable fact of life, while Barthes envisions it as 
opportunity for repeated penetrations of the original text. They concur that noise is inevitable. But Shannon wants to minimize noise, Barthes to maximize it. Similarly, Shannon regards redundancy as a necessary evil, whereas Barthes sees it as an erotic pleasure that swells a compact text into a gargantuan commentary.

A discipline is not, then, only an abstract field of inquiry. It implies a specific workplace and set of institutional affliliations, and these in turn imply community norms that invest concepts with values. Shannon was an electrical engineer who worked for the world's largest information conglomerate. At AT\&T, room in the channel translated directly into more expense for the company. Shannon's dedication to economy was reinforced by the multinational corporation that gave him a paycheck, as well as by the scientific community that gave him recognition. Consequently, he was not interested in just any economical explanation. He looked for one that would allow language itself to be more compressed. He did pioneering work on how to eliminate redundancy through proper coding (Shannon, I95I). His credo was compatible with the commercial economy within which he worked, as well as with the disciplinary economy that informed his aesthetic sensibility. Reduce and simplify, shorten and compress.

Barthes's approach is the opposite. Not only does he convert Balzac's I 3000-word short story into a 75000-word analysis; he also implies that there is no valid way to compress this expansive interpretation. He defines the five codes in an appendix, for example, indicating that in compressed form they are peripheral to his enterprise rather than its concentrated essence. This ideology of excess is not, of course, unconnected to his economic situation. He writes as a critic within a literary establishment, where fame, money, and power come from generating new words from old texts. The more texts are opened to accommodate his words and others, the more the community in which he works will reward him.

The different values Barthes and Shannon assign to equivocation can now be brought into clearer focus. Recall that Shannon defined equivocation as information that the message sender did not intend. From a commercial point of view this information is superfluous, since the company transmitting the message is unlikely to be reimbursed for it. Consequently, Shannon assumed that the equivocation 


\section{The Figure in the Carpet}

should be subtracted from the received message to get the original text back again. Barthes naturally disagrees, for if a text's meaning were limited to what the writer intended, the possibilities for literary criticism would be drastically curtailed. Since the canonized author is not paying for the message transmission, his intentions have no economic value. On the contrary, it is the consumers of Barthes's critical text who matter, and it is in their interest to see message noise increased, for then they are reassured that new and different books can be produced from the same canonized texts. Barthes therefore advocates that the equivocation should be added to the message rather than subtracted from it.

When Barthes contrasts rereading with consumption, he makes this difference in orientation explicit.

Rereading, an operation contrary to the commercial and ideological habits of our society, which would have us "throw away" the story once it has been consumed (or "devoured"), so that we can then move on to another story, buy another book, and which is tolerated only in certain marginal categories of readers (children, old people, and professors), rereading is here suggested at the outset, for it alone saves the text from repetition (those who fail to reread are obliged to read the same story everwhere).... Rereading is no longer consumption, but play. [1974: 15-16]

The emphasis on play is thus Barthes's answer to Shannon's ideology of use. If texts are useful, then they can be used up. Only when they are infinitely equivocal, forever supplementing their original message with noise supplied by the reader, are they saved from the capitalistic economy that would consign them to obsolescence.

But wait. The situation cannot be this simple. Surely there must be room within disciplinary economies for moves that run counter to the mainstream or that deflect it in a different direction. Otherwise disciplines would be much more homogeneous than we know they are, and they would be static over time, which we know they are not. I turn now to discuss a countermove within information theory which foreshadowed the shift to chaos theory. I also interrogate Barthes's reading more closely, seeing in it a complex strategy having as much to do with his individual desires as with the disciplinary economy within which these desires are inscribed. 
If Barthes had read Weaver's essay in The Mathematical Theory of Communication, he would have found the opposition sounding very much like him. Like Barthes, Weaver is a commentator. His essay originally appeared as an article in Scientific American and was intended to interpret Shannon's theory for a general scientific audience. Like Barthes, Weaver cannot resist expanding his role as commentator. He therefore speculates on how information theory might be extended beyond the strict engineering interpretation that Shannon gave it to include questions of semantics and behavior. As he leaves behind the terminology of use for the language of desire, his perspective undergoes a significant change. He suggests that into Shannon's diagram one might insert a box for "semantic noise," responsible for "perturbations or distortions of meaning which are not intended by the source but which inescapably affect the destination." This sounds conventional enough; however, he then goes on to say that "it is also possible to think of an adjustment of original message so that the sum of message meaning plus semantic noise is equal to the desired total message meaning for the destination" (Shannon and Weaver, I949: II6). Thus the "desired meaning" goes from being what the sender intended to whatever comes out at the end after semantic noise has been included.

With this shift Weaver comes very close to Barthes's position. S/Z is designed to increase semantic noise as much as possible, both in the interpretations it brings to bear on Balzac and in the extensive commentary it physically inserts between Balzac's message units. When Weaver suggests that desired meanings could result from the addition of semantic noise, his formulation is in sympathy with Barthes's project to increase noise as much as possible. Is it a coincidence that a commentator should envision information not intended by the sender as being also desirable, perhaps even more desirable than the authorized message? However we wish to read Weaver's flirtation with equivocation, it is clear that here, in a text bound together with Shannon's original articles and read widely throughout the discipline, is a voice expressing views at odds with the disciplinary economy. Thus individual desire is also a factor affecting the way theories are interpreted.

For Barthes, desire and economy are mutually reinforcing. Yet 
economic motives that have their bases in disciplinary infrastructures may still be shaped in distinct ways by individual desire. To see how this works in $S / Z$, consider how Barthes chooses to decode his "tutor text." He writes as if the codes he distinguishes are preexistent in the culture, in the text, or in both together. As Shannon could tell him, this is not true. Codes are a matter of choice. They may be chosen well or ill, but they are always chosen, not inherent in the message.

The code that carries the most interpretive weight in Barthes's reading is what he calls the "Hermeneutic Code," the "Voice of Truth." This code traces Sarrasine's simultaneous pursuit and evasion of the truth that Barthes situates at the center of Balzac's tale: that Zambinella, the singer with whom Sarrasine has fallen violently in love, is not a woman but a castrated man. Barthes's decoding suggests that the exchanges in Balzac's story proceed according to an economy of castration. In his reading, all the characters are located in relation to the phallus - whether they want one, pretend to have one, or want to take one away from someone else. Moreover, castration is presented as being highly contagious, so that by the end of the story virtually every character is presented as having caught it, even those who never had a penis to begin with. Barthes thus reads the story as a superstructure erected on the economic base of the phallus.

A very different way to read the story is implicit in Barbara Johnson's critique of Barthes's interpretation (Johnson, I980). She points out that Barthes supplies the word "castration"; Balzac's text never uses it. "Castration is what the story must, but cannot, say. But what Barthes does in his reading is to label those textual blanks 'taboo on the word castrato.' He fills in the textual blanks with a name. He erects castration into the meaning of the text, its ultimate signified" (p. Ir). In contrast to Barthes's focus on castration, Johnson's reading puts narcissism at the center of Balzac's story. In her view, Sarrasine's transgression consists not in coming into contact with the highly contagious disease of castration but in preferring an essentially male fantasy of what a woman is-a man without a penis - to the radical otherness of woman herself.

Johnson's reading makes clear that Barthes's decoding of Balzac's 
text is not merely a neutral report on what is there but a strategy to gain control of the text's meaning. Disclaiming any desire to control the text, to silence or neglect any of its multivalent voices, Barthes nevertheless centers it on a void for which he then supplies the name. Moreover, his claim that it is what has been added to Balzac's text that most satisfies desire resembles Sarrasine's narcissism. Deleting from Sarrasine's actions the egotism of which he too is guilty, Barthes gets the pleasure of a narcissistic correspondence between his interpretive strategy and Sarrasine's character, while at the same time interpreting both so as to conceal this common feature.

Barthes cagily avoids the question why he choses Sarrasine as his "tutor text," but Sarrasine is ideal for his purposes despite its realistic texture, for it has an absent center in the missing phallus. Because this center is already empty, Barthes's strategy of filling it as he desires can be hidden from notice. Using the same strategy, Barthes attributes to information a centrality and to his "noise" a marginality that imply he does not share in the will toward mastery he attributes to information theory. But when noise occupies center stage-as it surely does in $S / Z$ - this constituting difference between information and noise ceases to operate. ${ }^{12}$ When the center is empty-or has been emptied by discourse that claims to be marginal-there is no meaningful distinction between margin and center in terms of the power they exercise. Thus Barthes's reading of Balzac cannot be understood solely through its reinforcement by a disciplinary economy. Also important are individual desires and agendas.

Like ecology, then, economy is an incomplete explanation. Ecology limits what can be but does not determine what is; economy influences what will be heard and attended to but does not adequately explain why a message is presented in a particular way in a given text. Since equivocation has emerged as a pivotal term, it obviously signals an intersection of some importance. In the work of Michel Serres, equivocation appears in highly fissured and self-re-

\footnotetext{
'-The more general point is that deconstruction depends upon a rhetoric of marginality, which can be effective only when it is in a minority position. To the extent that deconstruction has become an accepted ideology within literary studies (if not, indeed, a majority position among publishing scholars), it is in the same position as Barthes in $S / Z$.
} 
flexive form, marking a site where the divergences and complicities among ecological constraints, economic infrastructures, and individual desires come strongly into play.

\section{Equivocation}

Of the literary theorists discussed in this chapter, Michel Serres is by far the most knowledgeable about the science of chaos. It is central to his project; there is scarcely a chapter of his extensive writing unconcerned with its implications. He uses it, however, in two different ways. In the first, the new paradigms provide him with an implicit vantage point from which to reassess classical concepts. Such a standpoint is necessary, he implies, because we still operate largely within the boundaries of classical thought. Frequently he chooses as sites for this reassessment moments when the classical paradigms were forged, such as the origin of geometry or the formulation of an ideal Platonic object. By showing what was excluded at these originary moments, he reveals the complicities that classical constructions share.

In the other mode, Serres attempts to extend the new paradigms to universal theories. Perhaps the best known is parasitism (The Parasite, 1982b). Playing on the three senses (in French) of the parasite-noise in a communication channel, an unwelcome guest, and an organism that feeds off its host-he uses its equivocal connotations to create a general theory of exchange. In my view the theory is not successful, for reasons I will indicate shortly. But I find it interesting that such a theory was attempted at all. Why would the theorist who warns us that "the global does not necessarily produce a local equivalent, and the local itself contains a law that does not always and everywhere reproduce the global" (I980: 75) want to create a global theory?

The problem is not peculiar to Serres. As we shall see in chapter 8 , it engages many contemporary theorists. I believe that it arises because the more intense the theorist's commitment is to the ideology of the local, the more he feels compelled to strengthen it by making it universally valid. This compulsion leads to a paradoxical attempt to extrapolate a general theory from paradigms that imply there are 
no general theories. In its paradoxical impulses as well as its subject matter, Serres's project is prototypical of attempts to extend chaos theory to a general theory of culture. Out of its strong internal tensions-one might almost say out of its turbulence-equivocation emerges as a central concept. As different voices compete within the channel of Serres's writing, equivocation serves both as the keystone for his theory of communication and as a metaphor for the conflicting impulses inherent in his interdisciplinary approach.

Serres's commitment to local knowledge is apparent in his essay "Platonic Dialogue" (1982a: 65-70). He points out that writing is inherently global, because it is "first and foremost a drawing, an ideogram, or a conventional graph" (p. 65), that is, a mark recognized as a form independent of its particular manifestation. Experienced readers are so accustomed to screening variations in letter formations from consciousness that they are only subliminally aware of them. But children learning to read frequently notice and comment upon the difference between a printed and a handwritten $a$, or between $t$ 's with curved and straight bottoms. To the adult reader, the child's focus on these variations is misguided; yet these "cacographies" (1982a: 66), as Serres calls them, are all that ever exist. In learning to suppress local variations, Serres implies, we also blind ourselves to the richly various actualities of the material world.

No less than writing, speech also depends upon suppressing the actual in favor of the ideal. Regional dialects, mispronunciations, stuttering, and slurring are routinely processed not as the sounds they actually are but as the idealized forms they represent. Thus "the first effort to make communication in a dialogue successful is isomorphic with the effort to render a form independent of its empirical realization" (p. 69). Through this demonstration, Serres opens a passage between the Platonic dialogues and the development of mathematics. What a Greek geometer meant by a circle, for example, was not the wavering form drawn in the sand but the abstraction toward which the mark was an imperfect gesture. Why, Serres asks, does mathematical discourse even today use the consensual "we" in demonstrations and proofs (p. 68)? Because mathematics is assumed to operate in a realm purged of all accidental, transitory features - that is, a space free of noise. Hence the Q.E.D.: "the dialectical method of the dialogue has its origins in the same regions as 
mathematical method, which, moreover, is also said to be dialectical" (p. 69). The argument's thrust up to this point is to show how thoroughly the suppression of noise has been incorporated into the foundations of modern thought.

From this demonstration Serres attempts to develop a general theory of communication. A dialogue, he asserts, "is a sort of game played by two interlocutors considered as united against the phenomena of interference and confusion. ... These interlocutors are in no way opposed. . . they battle together against noise. . . To hold a dialogue is to suppose a third man and seek to exclude him" (pp. 66-67). Although it is not clear why noise should be identified with a "third man," Serres apparently arrives at it by analogy with the excluded middle of binary logic. Just as classical mathematics developed by excluding a third possibility between true and false, so dialogues (and dialectics) evolved by excluding a "third man" who threatens to disrupt communication.

In privileging the "third man," Serres ostensibly proposes to deconstruct the traditional hierarchy of information and noise by inverting it and then revealing the existence of a hitherto unrecognized third term. This deconstructive thrust, however, gives way to the synthetic impulse to make the parasite into a universal concept in its own right. As Serres moves from reinscribing local knowledge to a synthetic theory of noise, conflation is the order of the day. In The Parasite, the "third man" becomes a general operator that applies indiscriminately to La Fontaine's fables, thermodynamically irreversible systems, communication channels, and economic exchanges. Such wide-ranging application is possible only because of metaphoric slippage in the half-numerical, half-anthropomorphic "third man." The "third man" can act as an operator in human exchange because it anthropomorphizes noise; it can explain why systems become more complex because it goes by the same name as the formal term that appears in information theory.

With such equivocal definitions, it is scarcely surprising that virtually any exchange can be explained in terms of the parasite. But what does such a theory tell us? Whatever else it intimates, the theory works (if it works) only by assimilating obvious differences into apparent similarities. And yet the explicit intention in creating it was 
to liberate the noise of empirical differences from the oppression of idealized similarities.

Given this paradox, it is instructive to see how Serres treats the suppression of difference when other people do it. In "Mathematics and Philosophy: What Thales Saw ..." (1982a: 84-97), Serres takes as his historical site Thales's discovery that he could determine the height of a pyramid by measuring its shadow. He imagines Thales standing in the Eygptian desert before one pyramid; two others loom in the distance, the same and yet somehow different. The mathematical concept of similarity, Serres suggests, is already encoded into the scene. Thales stands "in the domain of implicit knowledge" (p. 89); all he need do is make it explicit. Yet the movement from implicit to explicit knowledge is not inconsequential. It requires that the shadow be negated in its particularity and rendered as the mark of an idealized form. Thus at the birth of geometry, the "shadow of opinion, of empiricism, of objects" is sacrificed to "the sun of knowledge and of sameness" (p. 90).

The components of the scene, Serres notes, are the same as those in Plato's allegory of the cave: sun and shadow, explicit and implicit knowledge, idealized form and empirical fuzziness. No less than the allegory of the cave, the origin of geometry establishes "the scene of representation ... for Western thought for the next millennium" (p. 92). Only when this "immense historical cycle had finally come to an end" (p. 93) could non-Euclidean geometry and Cantor's "monstrous" sets enter the space of knowledge as legitimate subjects for inquiry. According to Serres, the shift is characterized by the displacement of a linear, chronological aesthetic based on a vision of time passing always at the same pace and measured in the same units by an aesthetic that focuses on spatial deformations and local turbulences. ${ }^{13}$

This line of thought suggests that spatial metaphors will be central to an understanding of the rhetorical moves that allow Serres to celebrate difference in his inscriptive mode while simultaneously

1:The influence of Mandelbrot is clear here. I suspect, however, that Serres may not really understand the mathematics, for fractal geometry has not renounced globalization by any means (a point discussed in chap. 8). 
suppressing it in his general theory. Generally speaking, space for Serres is identified with difference, whereas time connotes sameness. In Feux et signaux de brume (1975) he writes, "Time [is] the most immediate and simplest esthetic projection of ordered structure. With time, the esthetic is in order and those in political power are quite pleased. Spaces are repressed because they are possibly, better yet, certainly, disorderly. Reason, the political powers that be, prefer order rather than disorder, time rather than space, history rather than multiplicities" (p. I64). What metaphors does Serres use to liberate space from the "political powers that be"? More precisely, what metaphors allow him to negotiate between the drive toward synthesis in his own writing and the authorized voice that locates this impulse in external political authority?

One is the image of the passage, the journey negotiated with difficulty between narrow straits. In Hermes $V(1980)$ Serres appropriates this geographical terrain as a central metaphor for his project, especially for the passages of his writing:

No, the real is not cut up into regular patterns, it is sporadic. ... I am looking for the passage among these complicated cuttings. I believe, I see that the state of things consists of islands sown in archipelagoes on the noisy, poorly understood disorder of the sea. . . . Passages exist, I know, I have drawn some of them in certain works using certain operators. . . . But I cannot generalize, obstructions are manifest, and counterexamples abound. [1980: 23-24]

The mediating potential of this image is made clear in Serres's discussion of space in classical Greece (1982a: 39-53). "The Greek cities were dispersed, reciprocally closed insularities ... in which every man worthy of the name ... . was inside, while on the exterior of this political space animals, barbarians with growling languages, circulated in a chaotic multiplicity of sociopolitical spaces" (p. 5I). In this world, when connections are lost or passages not completed, no one "can speak any longer, and we have the irrational or the unspeakable-the incommunicable, to be very precise" (p. 50). The Greeks dedicated themselves to rationality, Serres implies, because universality was a hard-won accomplishment in their dispersed space. When one must fight to establish connections, one 
does not worry about the tyranny of globalizing theories or universal forms.

In contrast to the "sporadic" space of ancient Greece is the global village Serres inhabits, spanned by supersonic transport and bound by instantaneous satellite communication. Why does he assume this space is "in tatters"? Because the assumption is one of the rhetorical gestures that allow him to reconcile his globalizing impulses with his commitment to local knowledge. "Therefore I assume there are fluctuating tatters," he writes in a statement only apparently at odds with his attempt to build universal theories ( 1980 : 23). In fact, the assertion is what enables the theories to be advanced, for it is only when space is inherently and irrecoverably "tattered" that these theories are distinct from the repression of difference which the powers that be practice. For Serres to assume that his space is dispersed is as audacious as for the Greeks to assume that their space was continuous-and as necessary.

These divided impulses-the desire to proclaim space dispersed and the effort to assimilate difference into similarity-join in the image of the spiral. It is an appropriate image, for in its upward thrust it expresses the yearning for difference; then difference is assimilated into similarity when the trajectory conforms to a circular pattern; yet difference asserts itself again as the spiral continues upward, ad infinitum. Serres's essays are characteristically structured as spirals, coming around again and again to the same point displaced along an axis. For example, in "The Apparition of Hermes: Dom Juan" (I 982a: 3-I4), the same operators are recycled through slightly displaced locales in Molière's play. "The demonstration begins again" (p. 5), he writes as he considers the first act; "the same demonstration begins again" (p. 8), he repeats a few pages later; once more, the "demonstration begins again" (p. I3), until he finally arrives at patterns so pervasive that he can proclaim them to be "universal" (p. I I).

The classical text that Serres identifies as closest to his world view is Lucretius's De rerum natura. It is, he argues, structured like a spiral (r982a: 98-r 24). Moreover, it envisions the universe as a spiral. "Space and time are thrown here and there. There is no circle. But stochastically, turbulences appear in space and time. And the 
whole text creates turbulence ... the creative science of change and of circumstance is substituted for the physics of the fall, and of rigorous trains of events. Neither a straight line nor a circle: a spiral" (p. 99; emphasis added). The ideology of the local, never entirely absent in Serres's writing, is explicit in this essay. Had Lucretius's vision of the clinamen prevailed, the world might be dedicated to chaos rather than order, Venus rather than Mars, love rather than death. "The order of reasons is repetitive, and the train of thought that comes from it, infinitely iterative, is but a science of death. . . . Stable, unchanging, redundant, it recopies the same writing in the same atoms-letters. . . . Everything falls to zero, a complete lack of information, the nothingness of knowledge, nonexistence." But when the Lucretian clinamen enters the picture, it "cures the plague, breaks the chain of violence, interrupts the reign of the same, invents the new reason and the new law ... gives birth to nature as it really is" (p. 100). As we saw in chapter 4, turbulence cannot be adequately modeled through any simple form. Certainly it cannot be modeled as a spiral, which is far too orderly and predictable to express its extreme complexity. Serres does not so much express turbulence, then, as tame it when he envisions the world as a spiral. The strategy suggests that he may be more ambivalent about disorder than his rhetoric indicates.

This ambivalence is writ large in the contrast between the retrospective and globalizing modes in Serres's writing. Returning to sites where global theories were formed, he reinscribes the local knowledge they suppressed; but looking at the world locally, he cannot resist organizing scattered sites into global theories. I conjecture that Serres, despite his professed commitment to the local, keeps driving toward the universal because he cannot help feeling what virtually all scientists and very few poststructuralists do: the power of scientific explanations. His writing does not merely discuss literature and science. Informed both by the expanding economy of poststructualism and by the contracting aesthetic of science, it is literature-andscience. Taking equivocation as its central topos, it is also itself deeply equivocal, for the different voices of literature and science are both trying to occupy the same channel at the same time.

In "Lucretius: Science and Religion" (I 982a: 98-I24), Serres 
tackles the problem of his divided loyalties head on. Science, he acknowledges, is universal: "Two and two make four; heavy bodies fall, according to the law of gravity; entropy increases in a closed system, regardless of the latitude and whatever the ruling class." How can this universality be reconciled with a proposition he believes just as strongly, that "science is conditioned by postulates or by decisions that are generally social, cultural, or historical in nature?" How, in other words, can science be "conditioned but unconditional? No one has escaped this dilemma," Serres admits, certainly not himself (p. 106). He comes close to resolving the paradox when he argues that the truth content of science is a necessary but not sufficient condition to determine the final form of a scientific paradigm. Reality imposes constraints that cause some hypotheses to be verifiable, others not. But it does not determine which theories are actually realized or how they will be expressed. These things depend on cultural and historical conditions peculiar to the moment. With this lucid insight, Serres has what he needs to open the narrow straits of his passages into easily traversed terrain.

But he does not use it. Instead, the equivocation characteristic of his writing becomes more intense as it comes closer to potential resolution. Consider the sentence that contains the essential insight. It demonstrates in microcosm how intrinsic equivocation is to Serres's rhetoric. Musing on how science can be at once local and global, he writes, "In this case and a thousand like it, you can always proceed from the product to its conditions, but never from the conditions to the product" (p. I06). The generalizing impulse is apparent in "always" and "never"; yet these words are preceded by the equivocal phrase "in this case and a thousand like it," which envisions the global as a mass of localized points, not as a true universal. Equivocation is also apparent in the sense of the sentence, which could be paraphrased as "universals apply, but not always." To see whether they apply in a specific case, one has to consider the direction one travels. If one goes backward through time, one can "always" universalize; if forward, "never." Into which category does this statement fall? Since it spans both directions, it must be both true and untrue, local and universal, as its rhetoric signals. In other words, the sentence contains an undecidable proposition. At least since 
Russell and Whitehead, we have known how to analyze propositions of this kind. The sentence is undecidable because it is paradoxical, and it is paradoxical because it is self-referential. ${ }^{14}$

To identify the paradox, however, is not to resolve it. The statement is unavoidably self-referential, because Serres's entire project is caught between going backward in time when he reinscribes difference in classical paradigms and going forward in time as he strives to create a universal theory of local difference. This divided temporal impulse reflects his divided loyalties toward literature and science. To speak unequivocally would imply choosing one of these polarities over the other; and this he cannot do without vacating the site he has chosen for his project. Thus when he addresses the problem of divided loyalties directly, the prose characteristically becomes more infolded, for only so can the tensions that define the project's site remain intact.

Nowhere are these tensions more apparent than in "The Origin of Language: Biology, Information Theory, and Thermodynamics" (I982a: $7 \mathrm{I}-83$ ). In this essay Serres tries to expand informational equivocation into a general theory of language. After reviewing the second law of thermodynamics, he points out that fighting against entropic decay is the openness of all living systems-their ability to take in sunlight, food, information. Since this flow sustains life, he proposes that we regard ourselves not as stable bodies through which constantly changing streams of matter and energy flow but as stable flows encased within constantly changing bodies. Consider an ocean wave: although the water appears to flow, a typical water molecule moves only a few feet during its entire lifetime. The shores, by contrast, are eroding every day. In an elegant reformulation of Heraclitus, Serres writes, "One always swims in the same river, one never sits down on the same bank" (p. 74).$^{15}$ This part of the essay is

\footnotetext{
${ }^{14}$ Serres's views on this matter are very similar to those of Lem, who also positions himself at an interstice between literature and science. It is no accident that Lem, like Serres, sometimes opts for a transcendent viewpoint rather than a self-reflexive loop. His topological model, for example, must of necessity be one of the shapes possible within postmodern culture. But Lem does not inquire into the conditions of possibility that authorize his topological model of culture, preferring to posit it as a transcendent concept rather than as a model that models itself.

${ }^{15}$ Serres may not be quite correct here. It is true that waves in an ocean serve to transfer energy rather than matter, but I rather think that water molecules in rivers
} 
in the retrospective mode, an attempt to insert into traditional paradigms the empirical differences they suppress.

Having allowed the voice of difference to speak, Serres then moves to assimilate it into a general theory of language. His inspiration is the article by Henri Atlan (I 974) cited in chapter 2, in which Atlan shows that the value of the equivocation in Shannon's equations depends upon where the observer is positioned in relation to the message. If the observer knows what the "correct" message isthat is, if she is positioned at the message source-the equivocation will appear as unwanted noise that should be subtracted from the message's original information. If the observer is positioned outside the system, however, she is in a position to notice the effect of the equivocation on the system as a whole. She thus can see instances in which the equivocation causes the system to reorganize itself at a higher level of complexity. In these instances, Atlan argues, the equivocation should be given a positive sign, for it has in effect become information. It is not hard to see why this argument would appeal to Serres (to say nothing of Barthes, who would find it deliciously redundant). Serres correctly sees in Atlan's article scientific validation for his view of noise as a positive force.

However, he continues to a conclusion found in none of his scientific sources. He apparently arrived at it by conflating Atlan's article with François Jacob's speculation (I 976) that biological organisms are structured in interlocking levels of integration, like so many Russian dolls stacked inside one another. If organisms are structured in hierarchical levels, Serres reasons, then one can conclude that the value of the equivocation changes with each level, so that each higher level functions as a "rectifier of noise" for the level beneath ( $982 \mathrm{a}: 78$ ). If this were so, noise at a lower level would always be transformed into information at the next higher level, and organisms would be unified only because they are fragmented-an implication Serres expands into the paradoxical conclusion that language itself originates in noise.

At this point, his divergence from his scientific sources is clear.

do move. Otherwise, as a colleague pointed out, why would mountain streams run dry? 
There is no scientific evidence that noise is rectified from level to level within human beings. Nor do these theories say that noise necessarily becomes useful information. Whether noise will have a positive or negative effect on systemic organization depends on the stability of the system, the kind of feedback loops at work, the amount and kind of noise injected, and when the injection occurs (Atlan, 1974; Jacob, 1973; Nicolis and Prigogine, 1977). Serres's claim that the rectification of noise "is valid for all levels" and is a universal "law of the series" is simply not true.

For Serres, however, this conclusion is merely the springboard for further speculation. He links equivocation with the Freudian unconscious, reasoning that if noise can be either positive or negative, then one can say that "in one case, it covers up; in the other, it expresses." Thus he concludes that the "entire symbolic function is embedded in this process, the entire strategy of free assocication, Freudian slips, jokes and puns" (p. 80). As he diverges further from fact, his rhetoric becomes more insistent that his theory is scientific. "These matters are straightforward" (p. 78), he claims, alleging that "we can speak about [a layered unconscious] by using a discourse which ultimately can be expressed in mathematical terms" (p. 79). To whom does the "we" in this sentence refer? Not to the sources Serres cites-Henri Atlan, François Jacob, and Claude Shannon, for example-or to any other scientist of whom I am aware.

If we think about what is happening at this point in Serres's argument, we can see that he is trying to make the rectification of noise into a principle that applies equally to language, human psychology, the universe, everything. The irony is that in transforming equivocation in this way Serres has made it univocal, for it leads always to increasing order. Thus "rectified," equivocation is at least as capable of suppressing difference as any of the Platonic concepts Serres addresses. Projecting this no-longer-equivocal equivocation onto an imagined scientific "we," he dreams that it can fuse the deep shadows of the unconscious with the clear sun of mathematical equations, just as he dreams that his own discourse is at one with all other discourses, from Plato to Freud to Shannon. Of course these dreams are not realities. But they nevertheless represent a culminating vision, as the heightened language signals, for at last the noise of 
difference has been made to speak the language of unity. Serres imagines that through his theory we will finally understand the "packages of chance" that come "crashing at our feet, like the surf at the edge of the beach, in the forms of eros and death" (p. 80). It is from this aporia that his theory of the parasite emerges, traversing a path that can be negotiated only in the equivocal language of dreams. ${ }^{16}$

In one sense Serres is a man ahead of his time, for he understands that the reevaluation of chaos within contemporary paradigms is a cultural shift of the first importance. It signals not just a new scientific or literary theory but a shift in the ground of representation itself. However, the passage between wacky theorizing and brilliant insight in his writing is so narrow that it is sometimes hard to say on which side it falls. It seems most uncontrolled when it takes itself as subject, that is, when it operates upon the equivocation that is its abiding subject and underlying dynamic.

Then the strain of self-reflexivity, which I noted earlier as an undertone, is exaggerated until the prose is caught in the recursive loops typical of positive feedback, oscillating more and more wildly between the polarities that define its dialectic, insisting all the while that this oscillation is really unity. Self-referential paradoxes are, of course, the stuff of which deconstructive analyses and postmodern literature are made. Within the context of Serres's writing, however, they have the effect of conflating the distinct voices of literature and science into a cacography of confused claims. This transformation of equivocation into cacography signals failure, for Serres's project depends upon speaking both the claims of science to represent the world and the admission of language that it can represent only itself.

"At the end of The Parasite, Serres makes the significant gesture of rejecting the ocean-born Venus for the ocean itself. The gesture marks a new phase in Serres's development, in which he increasingly abandons all forms of order to crawl down the "manholes" or "bubbles" in the foam to commune with aboriginal chaos. These aspects of Serres's more recent work are discussed by Eric White in "Negentropy, Noise, and Emancipatory Thought" and by Maria Assad in "Michel Serres: In Search of a Tropography," both in Chaos and Order: Complex Dynamics in Literature and Science, ed. N. Katherine Hayles (Princeton: Princeton University Press, forthcoming). 
208 The Figure in the Carpet

In attempting to articulate literature and science together through the three modes of interaction discussed in this chapter-ecology, economy, and equivocation-I have sought to create an equivocal site at which both disciplines can have a voice. The vision I hope to have conveyed is not of science influencing literature but of literature and science as two mingled voices within the cacophonography that we call postmodern culture. 